\title{
Adjunct use of mouth rinses with a sonic toothbrush accelerates the detachment of a Streptococcus mutans biofilm: an in vitro study
}

Tatsuya Ohsumi ( $\boldsymbol{D}$ osumi@dent.niigata-u.ac.jp )

Niigata University https://orcid.org/0000-0003-3970-8332

Shoji Takenaka

Niigata Daigaku Shigakubu

Yuuki Sakaue

Niigata University

Yuki Suzuki

Niigata University

Ryoko Nagata

Niigata University

Taisuke Hasegawa

Niigata University

Hayato Ohshima

Niigata University

Yutaka Terao

Niigata University

Yuichiro Noiri

Niigata University

Research article

Keywords: Biofilm, Streptococcus mutans, mouth rinse, detachment, sonic toothbrush

Posted Date: April 15th, 2020

DOI: https://doi.org/10.21203/rs.2.22750/v2

License: (c) (i) This work is licensed under a Creative Commons Attribution 4.0 International License.

Read Full License

Version of Record: A version of this preprint was published at BMC Oral Health on June 3rd, 2020. See the published version at https://doi.org/10.1186/s12903-020-01144-0. 


\section{Abstract}

Background: The aim of this in vitro study was to examine the possible enhancement of the biofilm peeling effect of a sonic toothbrush following the use of an antimicrobial mouth rinse.

Methods: The biofilm at a noncontact site in the interdental area was treated by sound wave convection with the test solution or by immersion in the solution. The biofilm peeling effect was evaluated by determining the bacterial counts and performing morphological observations. A Streptococcus mutans biofilm was allowed to develop on composite resin discs by cultivation with stirring at $50 \mathrm{rpm}$ for 72 hours. The specimens were then placed in recesses located between plastic teeth and divided into an immersion group and a combination group. The immersion group was treated with phosphate buffer, Peridex ${ }^{\mathrm{TM}}$ mouth rinse or Listerine ${ }^{\circledR}$ mouth rinse. The combination group was treated with Peridex ${ }^{\mathrm{TM}}$ or Listerine ${ }^{\circledR}$ and a sonic toothbrush.

Results: The biofilm thickness was reduced by approximately one-half compared with the control group. The combination treatment produced a 1 log reduction in the number of bacteria compared to the Listerine ${ }^{\circledR}$ immersion treatment. No significant difference was observed in the biofilm peeling effect of the immersion group compared to the control group.

Conclusions: The combined use of a sonic toothbrush and a mouth rinse enhanced the peeling of the biofilm that proliferates in places that are difficult to reach using mechanical stress.

\section{Background}

Dental biofilm control is the most important method of preventing dental caries and periodontal disease. Toothbrushing plays a central role in self-care. Sonic toothbrushes have been reported to remove dental biofilm better than manual toothbrushes [1-3]. Sonic toothbrushes have been developed to improve and promote oral hygiene [4], and have been widely promoted as a tool that easily and effectively removes dental biofilm [4]. In addition, electric toothbrush is beneficial in maintaining the oral health of patients with neuromuscular disorders and reducing the burden of caregivers in completing oral care. Electric toothbrushes have been shown to be an effective adjunct method for plaque control, particularly in patients with a low dexterity level [5]. Auxiliary cleaning instruments must be used to control dental biofilm in the areas where the sonic toothbrush is unable to directly contact the dental surface (e.g., the interproximal area), even though sonic vibrations. The presence of a residual dental biofilm in the interdental areas remains a problem $[6,7]$.

Various methods have been used to clean the interdental area, such as toothpicks, dental floss, and interdental brushes. Among these options, the interdental toothbrush is considered the most effective method $[8,9]$. However, some people experience difficulty using auxiliary cleaning tools, such as interdental toothbrushes, particularly in between the molars. Noncompliance with interdental cleaning is a key issue in self-care, as inadequate compliance leads to periodontal disease $[10,11]$. Generally, clinicians postulate that self-care will be more effective if the interdental biofilm is removed with a sonic 
toothbrush. Dental biofilm removal by sonic toothbrushes at non-contact sites has been evaluated in some studies $[12,13,14]$. Even when a sonic toothbrush is used like a manual toothbrush, the complete removal of the dental biofilm in noncontact areas is difficult to achieve.

A previous study investigated the effect of the shear stress of the water current generated by a sonic toothbrush on dental biofilm removal [12]. According to that study, high speed bristle motion creates turbulence in the oral cavity that produces shear stress parallel to the tooth surface. The shear stress may remove the dental biofilm in areas not in direct dental contact with the brush $[15,16]$.

According to another study, dental biofilm removal at interproximal sites that are not in direct contact with the sonic vibrations from the toothbrush is limited [17]. A mouth rinse represents a chemical control method that can be used as an auxiliary tool to address this deficiency. As shown in our previous study, penetration and disinfection are obtained with mouth rinse alone, but the biofilm structure is not completely removed [18]. Streptococcus mutans produces acids from sugar metabolism and uses sucrose to synthesize extracellular polysaccharides (EPS). EPS is a glycan primarily involved in the development and protection of dental biofilms. Antimicrobial compounds have been shown to not function as intended [19]. This phenomenon can be explained by the degradation or delayed penetration by extracellular macromolecules (EPS) in biofilms. In other words, the biofilm cannot be removed only by immersion in the mouthwash. In one study, there was a report that the effect of chlorhexidine on biofilms caused a concentration gradient in the antimicrobial component, which was below the minimum inhibitory concentration and enhanced biofil formation [20]. When a dental biofilm is disinfected with a mouth rinse, its structure remains at the dental adhesion interface and may promote new bacterial adhesion [21]. The combination of the mechanical stress from the toothbrush and an antimicrobial mouth rinse exerts a synergistic effect on biofilm dispersal. This in vitro study examined whether an antimicrobial mouth rinse enhances the biofilm peeling effect of a sonic toothbrush. Namely, the sonic brush was used when a small amount of the mouthwash was present in the mouth, or after the application of the mouthwash that filled the interdental portion. An interdental Streptococcus mutans biofilm model was used to evaluate the removal of a dental biofilm by a sonic toothbrush in places where the toothbrush does engage in direct dental contact.

\section{Methods}

\section{Saliva collection and processing}

A sterilized saliva solution was prepared using a previously described method [22]. Unstimulated saliva was collected from a healthy person (one of the authors) who had not eaten, drank, or brushed for at least 2 hours prior to saliva collection. Saliva samples were diluted (1:10) with sterilized Ringer's solution containing $0.05 \%$ cysteine (Sigma-Aldrich, St. Louis, MO). Diluted solutions were centrifuged at 2,000 g for 10 minutes to remove any debris, and the supernatants were filter sterilized. The saliva solution was used to coat discs with salivary pellicles $[18,23]$. All study protocols were approved by the Ethics Committee for Clinical Research of Niigata University (approval no. 2019-0002). 


\section{Preparation of the biofilm structure}

S. mutans ATCC 25175 , which was originally isolated from carious dentin, was purchased from the American Type Culture Collection and was cultured anaerobically at $37^{\circ} \mathrm{C}$ on brain heart infusion (BHI) (Difco Laboratories, Detroit, MI) agar plates. Single colonies were selected, inoculated in BHI broth without sucrose, and incubated overnight at $37^{\circ} \mathrm{C}$ under anaerobic conditions. The preculture was transferred to $10 \mathrm{ml}$ of fresh $\mathrm{BHI}$ broth containing $0.5 \%$ sucrose under anaerobic conditions and cultured for 4 hours at $37^{\circ} \mathrm{C}$ under aerobic conditions. The absorbance of all bacterial suspensions at $600 \mathrm{~nm}$ was adjusted to 0.05 prior to inoculation.

Composite resin materials (Premise Flowable, Kerr, Orange, CA) were used as the attachment site for the biofilm structure. Standardized discs, $6 \mathrm{~mm}$ in diameter and $1.5 \mathrm{~mm}$ in thickness, were prepared and polished with 4000 grit waterproof silicon carbide paper; then, they were subjected to ethylene oxide gas sterilization for 4 hours. The discs were coated with $10 \%$ conditioned sterile saliva for 2 hours at room temperature. Biofilm structures were prepared as previously described [21]. S. mutans biofilms were allowed to form on the discs using a rotating-disc reactor (RDR) (Biosurface Technologies Corp., Bozeman, MT). This system is depicted in Fig. 1 and has been previously described in detail [24].

The discs were incubated for 90 minutes at $37^{\circ} \mathrm{C}$ in the $\mathrm{BHI}$ broth without sucrose, containing the $S$. mutans cell suspension while stirring at $75 \mathrm{rpm}$ to achieve initial adhesion. Following the adhesion phase, the stir disc was gently rinsed with $100 \mathrm{ml}$ of phosphate buffer $\left(5.0 \mathrm{~g} \mathrm{l}^{-1} \mathrm{NaCl}\right.$ and $2.5 \mathrm{~g} \mathrm{l}^{-1}$ $\mathrm{Na}_{2} \mathrm{HPO}_{4}, \mathrm{pH} 7.4$ ), and was aseptically transferred to a sterile reactor vessel filled with $300 \mathrm{ml}$ of diluted BHI broth (1:10) containing $0.05 \%$ sucrose. The biofilm was allowed to form for 72 hours while the solution was stirred at $50 \mathrm{rpm}$ under continuous flow aerobic conditions at a rate of $4.6 \mathrm{ml} \mathrm{min}{ }^{-1}$ during an incubation at $37^{\circ} \mathrm{C}$. The medium was changed every 12 hours. After the fixed incubation period, the rotating wheel was aseptically removed, and the specimens were washed three times with phosphate buffer.

\section{Treatment}

A pair of resin composite discs containing biofilms was inserted into recesses located between plastic teeth (Fig. 2A). These plastic teeth were then placed into a typodont model (Nissin Dental Products, Inc., Kyoto, Japan) located inside an exposure chamber containing either phosphate buffer or mouth rinses. The specimens were divided into six groups. Two independent experiments were performed to obtain $\mathrm{n}=$ 7 specimens per group. Each experiment consisted of an immersion group and a combination group. The immersion groups were treated with phosphate buffer (group C), a chlorhexidine gluconate Peridex ${ }^{\mathrm{TM}}$ alcohol-containing (11.6\%) mouth rinse (3M ESPE, USA) (group G), or a Listerine ${ }^{\circledR}$ Fresh Mint alcoholcontaining (21.6\%) mouth rinse (Johnson \& Johnson, USA) (group L). The combination groups received the test mouth rinses and treatment with a sonic toothbrush (designated in the groups as ST) (Philips Sonicare Flexcare HX6930; ST). The combination groups were designated as the $\mathrm{C}+\mathrm{ST}$ group, the $\mathrm{G}+$ ST group, and the L + ST group. 
In the immersion groups, the biofilm constructs were immersed in the tested solution for 5 seconds. In the ST combined groups, the biofilms were treated with a sonic toothbrush for 5 seconds at a position located $2 \mathrm{~mm}$ away from the disc (Fig. 2B-C). Thereafter, the discs with biofilms were gently rinsed with sterile phosphate buffer three times to remove excess treatments, and the residual biofilm structure was observed with a scanning electron microscope (SEM). In addition, the biofilm was recovered by an ultrasonic treatment, the viable cell count was estimated using the colony counting method, and the total number of bacteria was calculated using the PCR-Invader method.

\section{SEM imaging}

After treatment, the biofilm structure was observed with a SEM (EPMA-1610, Shimadzu, Kyoto, Japan). Specimens were washed with phosphate buffer and fixed with $2.5 \%$ glutaraldehyde for 2 hours. After fixation, the fixed specimens were dehydrated using a series of ethanol solutions ( 10 minutes each in 60, $70,80,90,95$, and $100 \%$ ethanol) and then air dried. The samples were sputtered with gold-palladium and examined using the SEM.

\section{Cryo-embedding, cryo-sectioning, and measurement of the thickness of the $S$. mutans biofilms}

The treated biofilm samples were embedded in a protective medium (Tissue-Tek O.C.T. compound, Sakura Finetek, Tokyo, Japan) as previously described [25]. The resin discs were placed on dry ice, and the medium was gently poured on them from above and allowed to freeze. The resin discs were peeled away from the embedded medium to ensure that the biofilm remained attached to the medium side. Then, the biofilm was placed on dry ice with the embedded side down. The embedding medium was used to cover the bottom surface of the exposed biofilm. The embedded biofilm samples were sectioned into 8 $\mu \mathrm{m}$ cross sections using a cryostat (CM 3050 S; Leica, Nussloch, Germany). The thickness of the biofilm was measured and partitioned into 10 section intervals. Twenty-five sections from each embedded sample were analyzed.

\section{Quantitative analysis of viable and total cells}

Colony counts and the PCR-Invader assay were used to quantify the numbers of viable and total bacteria in the test samples, respectively. Samples were washed three times with phosphate buffer and immersed in $3 \mathrm{ml}$ of phosphate buffer. Biofilms were collected by vortex mixing for 3 minutes, followed by ultrasonication for 5 minutes. Samples were serially diluted in autoclaved distilled water, and $100 \mathrm{ml}$ of each dilution were plated on $\mathrm{BHI}$ agar. The plates were incubated anaerobically for 48 hours at $37^{\circ} \mathrm{C}$, after which the number of viable colonies were counted.

The total number of bacteria was determined using the modified Invader PLUS method developed by BML Inc. (Saitama, Japan). The details of the PCR-Invader assay have been previously reported [26]. The PCR-Invader assay combines polymerase chain reaction amplification and invader detection, and can calculate the total number of bacteria with high sensitivity and speed. The primers for $S$. mutans were based on a region of the $16 \mathrm{~S}$ ribosomal RNA sequences. Bacterial DNA was extracted using Pure LC 
(Roche, Tokyo, Japan) and a MagNA Pure LC Total Nucleic Acid Isolation Kit (Roche). The template DNA $(3 \mathrm{ml}$ ) was added to $12 \mathrm{ml}$ of a reaction mixture containing $20 \mathrm{mM}$ primers, $2.5 \mathrm{mM}$ dNTP, $2.5 \mathrm{U}$ of AmpliTaq gold, $3.5 \mathrm{mM}$ primary probe, $0.35 \mathrm{mM}$ Invader oligo, and the Invader core reagent kit, which consisted of FRST mix and enzyme/ $\mathrm{MgCl}_{2}$ solution (F-primer, 5'-GGATTCGCTAGTAATCG-3'; R-primer, 5'TACCTTGTTACGACTT-3'; Tb-Primary probe, 5'-CGCGCCGAGGCCGGGAACGTATTCACC-3'; and Tb-Invader oligo, 5'-TGACGGGCGGTGTGTACAAGGCA-3'). Reaction mixtures were preheated at $95^{\circ} \mathrm{C}$ for 20 minutes, and then a two-step PCR was performed for 35 cycles $\left(95^{\circ} \mathrm{C}\right.$ for 1 second and $63^{\circ} \mathrm{C}$ for 1 minute) using the ABI PRISM 7900 sequence detection system (Applied Biosystems, Foster City, CA). Fluorescence values for carboxyfluorescein (wavelength/bandwidth: excitation 485/20 nm; emission 530/25 nm) were measured at the end of the incubation/extension step at $63^{\circ} \mathrm{C}$ for each cycle. Each assay was performed in triplicate and the mean values from the six independent samples were determined.

\section{Statistical analysis}

Statistical analyses were performed using the SPSS 11.0 (IBM, Armonk, NY) program. When applicable, data are presented as means \pm standard deviations (SD). The significance of measurement biofilm thickness, viable and total cell counts were determined using Kruskal-Wallis test and the post hoc SteelDwass test.

\section{Results}

\section{SEM observations}

SEM images of high and low cell density areas were captured from each group (Fig. 3). No apparent difference in the cell density areas was observed between group $C$ and the $C+S T$ and the $G+S T$ groups. On the other hand, the biofilm structure in the cell density area remaining on the disc tended to be reduced in the $L+S T$ group. A decrease in the number of bacteria was observed in the $L+S T$ group after sonic toothbrush treatment in areas with high and low cell density compared to the other groups. In the sparse areas, the biofilm exhibited a monolayer structure, and the intercellular density tended to decrease in the following group order: $\mathrm{C}, \mathrm{C}+\mathrm{ST}, \mathrm{G}+\mathrm{ST}$, and L + ST (Fig. 3, lower row).

\section{Biofilm thickness}

Using the frozen longitudinal sections, the biofilm height after the experimental treatments was measured. The maximum thickness of the residual biofilm structure that developed in this study was approximately $30 \mu \mathrm{m}$ for the control. The images of cryo-sections (Fig. 4) provided a higher resolution of the deeper layer. The thickness of the $L+S T$ group was significantly less than the control groups and the $C+S T$ groups ( $p<0.05$ for the $L+S T$ group).

\section{Quantitative analysis of the numbers of viable and total cells}


The number of viable cells (Log CFU $\pm \mathrm{SD} / \mathrm{ml}$ ) was $6.20 \pm 0.59$ (Group C), $5.89 \pm 0.83$ (Group G), $5.89 \pm$ 0.89 (Group L), $6.12 \pm 0.30$ (C + ST Group), $5.15 \pm 0.39$ (G + ST Group), and $4.69 \pm 0.38$ (L + ST Group). A significant reduction in the number of viable cells was observed in the $L+S T$ group compared with all the other groups ( $p<0.01$ for the $C$ and L groups; $p<0.05$ for the $C+S T$ group). A significant difference was not observed between the $C, L$ and $C+S T$ groups ( $p>0.05$ ) (Fig. 5). The total number of bacteria ( $\mathrm{Log}$ $\mathrm{CFU} \pm \mathrm{SD} / \mathrm{ml}$ ) was $5.72 \pm 0.11$ (Group C), $5.38 \pm 0.23$ (Group G), $5.97 \pm 0.19$ (Group L), $5.68 \pm 0.18$ (C+ ST Group), $5.20 \pm 0.24$ ( $\mathrm{G}+\mathrm{ST}$ group), and $4.79 \pm 0.11$ ( $\mathrm{L}+\mathrm{ST}$ group). The total number of bacteria was significantly reduced in the $L+S T$ group $(p<0.05)$ compared with all the other groups. A significant difference was not observed between the $C, L$, and $C+S T$ groups $(p>0.05)$ (Fig. 6).

\section{Discussion}

A quantitative analysis of the viability of bacteria present in dental biofilms was performed after various treatments with a mouth rinse and a sonic toothbrush. Hydrodynamic phenomena and microbiological aspects of the biofilm were also studied.

After the usual brushing, a mouth rinse was used as an adjunct. The water flow generated by the sonic toothbrush was carefully monitored to increase the effectiveness of the mouth rinse. The sonic toothbrush produces a liquid flow to the interdental area, and a fluid shear stress removes the biofilm [13]. Therefore, we hypothesized that the fluid shear stress, mouth rinse penetration, and bactericidal effect would combine to produce a synergistic effect when a mouth rinse containing an antimicrobial component was used. Although the biofilm was not eliminated from the dental surface, our results conform to the general consensus that the sonic toothbrush, namely, a toothbrush with bilateral symmetry and multidimensional and sonic action, enables biofilm removal in non-contact areas $[27,28]$. According to the literature, a more pronounced reduction in the biofilm is observed after the use of a sideto-side toothbrush than after the use of a toothbrush with multidimensional action [29, 30, 31, 32]. However, the application of these findings to a type of electric toothbrush that was not analyzed in this study should be avoided. In addition, since the present study focused on biofilm exfoliation, the altered distribution of viable and dead biofilm-forming bacteria after non-contact brushing treatment was not verified.

A sonic toothbrush generates microbubbles in the surrounding liquid, which is called cavitation. These bubbles contact the biofilm, creating shear stress that results in peeling of the dental biofilm [13], namely, removing the biofilm from areas that are not receiving direct contact from the bristles.

The mechanism by which the biofilm is peeled off is through shear stress. Some biofilms that have grown to a certain height exhibit physical smoothness due to saliva, food, and other materials that diffuse out from the surface layer. Shear stress does not remove biofilm from the interdental areas, allowing the biofilm to accumulate. An in vitro model in which a dental biofilm was deposited in the interdental areas was constructed to mimic this effect. 
As mentioned above, sonic toothbrushes generate bubbles in connection with increased liquid flow. Shear stress is exerted by these bubbles passing through the interdental areas [29]. We hypothesized that the combination of the sonic vibration of the sonic toothbrush with the antibacterial action of the mouth rinse would improve the removal of the dental biofilm removal compared to the use of the toothbrush alone. Synergistic effects were expected for the use of chemical mouth rinses in combination with mechanical toothbrushing.

As a method for further enhancing the biofilm removal effect of a sonic toothbrush and mouth rinse, a surface coating agent is applied to suppress the adhesion of bacteria to the teeth. By suppressing adhesion or reducing the adhesion capability, mechanical removal is facilitated. This coating would allow the shear stress of the water stream from the sonic toothbrush to more easily peel off any attached biofilm [33].

In the present artificial biofilm model, even when using Listerine ${ }^{\circledR}$, chlorhexidine, and the sonic toothbrush, significant biofilm peeling was not achieved with only 5 seconds of toothbrush action. S. mutans adheres firmly to the interface by producing sticky glucans $[34,35]$. The shear stress generated by the water flow created by the sonic brush exerts a peeling effect on the biofilm attached to the interface [28]. No significant difference was observed. However, in the presence of CHX or Listerine ${ }^{\circledR}$ mouthwash, the release effect was significantly improved. In addition, no significant difference was observed in the bactericidal effect of antibacterial components in the mouthwash. This lack of a difference is thought to be due to the fact that glucan, a constituent of the biofilm of $S$. mutans, impedes the penetration of antibacterial components and diminished the effect. We surmised that the bactericidal action was not substantially affected because of the short application time of 5 seconds. However, the biofilm peeling effect on the adjacent surfaces where the biofilm is difficult to mechanically remove was enhanced using the combination of the mouth rinse and the sonic toothbrush. Given the daily use of sonic toothbrushes, the application of the toothbrush to the same place for long periods is not realistic. Although 5 seconds is a relatively short action time, the peeling of the biofilm was significantly enhanced when the sonic toothbrush was used in combination with the mouth rinse. A comparison of the two mouth rinses revealed that Listerine ${ }^{\circledR}$ exerted a greater peeling effect than chlorhexidine. A potential explanation for this finding is a difference in the penetration rate of the antimicrobial component of the mouth rinse [36]. In the report by Wakamatsu et al., the permeation rate of the antimicrobial component of Listerine ${ }^{\circledR}$ was significantly higher in a 30 seconds comparison. Listerine ${ }^{\circledR}$ penetration was faster than $\mathrm{CHX}$, but shows how difficult it is for antibiotics to penetrate oral biofilms for a short time [18]. This result was similar to the result of this experiment because no difference was observed in CFU of the group that was only immersed. In contrast, the peeling action of the mouth rinse combined with a sonic brush is presumed to be mediated by a weakening of the binding force of the biofilm matrix by the mouthwash.

As countermeasures against oral biofilms, chemical control methods using antibacterial ingredients are applied in anticipation of enhancing the mechanical effect of toothbrushing. Due to the cavitation effect, the dental biofilm outside the range of the sonic toothbrush bristles may also be removed by the water 
current, if the biofilm adhesion is weak. The treatment and prevention of periodontal disease requires daily dental biofilm removal. Failure to comply with oral hygiene measures is frequently observed, particularly in difficult to reach areas, such as interdental areas $[37,38]$. If the sonic toothbrush effectively removes dental biofilm without making bristle contact, oral hygiene will improve. However, evidence supporting this hypothesis is currently unavailable in clinical settings. After comparing the in vitro data from the present study, the effects of brushing protocols on biofilm formation must be considered.

The difference between an oral biofilm and the $S$. mutans biofilm model used in this study should be taken into account. Clinical phenomena are not necessarily reproduced using a single bacterial strain. Although $S$. mutans is not representative of the complex group of microorganisms typically detected in dental biofilm, $S$. mutans easily forms biofilms and has been used as a model in various biofilm studies $[39,40]$. The glucan-containing extracellular polysaccharide produced by $S$. mutans strengthens the biofilm, increasing the difficulty of removal by brushing. Therefore, in future studies, we plan to combine the application of the sonic toothbrush with an enzyme that decomposes extracellular polysaccharides.

\section{Conclusion}

Listerine ${ }^{\circledR}$ and chlorhexidine mouth rinses were used in combination with a sonic toothbrush to evaluate dental biofilm removal from an in vitro model. The combined effect of the sonic toothbrush and the mouth rinse enhanced biofilm peeling from the adjacent dental surfaces that the toothbrush is unable to reach directly. Individual deficiencies in the chemical and mechanical removal methods are addressed by the synergistic combination.

\section{Abbreviations}

CHX: Chlorhexidine digluconate solution(s)

BHI: brain heart infusion

RDR: rotating-disc reactor

group C: immersion groups were treated with phosphate buffer

group G: immersion groups were treated with a chlorhexidine gluconate Peridex ${ }^{\text {TM }}$ mouth rinse

group L: immersion groups were treated with a Listerine ${ }^{\circledR}$ mouth rinse

ST: sonic toothbrush

SEM: scanning electron microscope

SD: standard deviations 


\section{Declarations}

\section{Ethics approval and consent to participate}

Ethics approval for the study was obtained from the Ethics Committee for Clinical Research of Niigata University approved this study (approval no. 2019-0002). Written informed consent to participate in the study was obtained from the participant.

\section{Consent for publication}

Not applicable.

\section{Availability of data and materials}

The datasets used and analyzed during the current study are available from the corresponding author on reasonable request.

\section{Competing interests}

The authors declare that they have no competing interests.

\section{Funding}

This investigation was supported in part by a Grant-in-Aid for Scientific Research (C) (no. 23592795) from the Japan Society for the Promotion of Science (JSPS) and a Grant-in-Aid for Young Scientists (B) (no. 16K20451) from the JSPS. The funding body played no role in the study design; data collection, analysis, and interpretation; and manuscript writing.

\section{Authors' contributions}

Conceived and designed the experiments: TO ST YN. Performed the experiments: TO ST YSA YSU RN TH. Analyzed the data: ST HO YT. Contributed reagents/materials/analysis tools: TO ST HO YT. Wrote the manuscript: TO ST. Performed the final review of the manuscript: YT YN. All authors read and approved the final version of this manuscript.

\section{Acknowledgments}

We thank Masayoshi Kobayashi for providing technical assistance with SEM analyses.

\section{Author information}

Affiliations

Division of Cariology, Operative Dentistry and Endodontics, Niigata University Graduate School of Medical and Dental Sciences, 2-5274, Gakkocho-dori, Chuo-ku, Niigata, 951-8514, Japan 
Tatsuya Ohsumi, Shoji Takenaka, Yuuki Sakaue, Yuki Suzuki, Ryoko Nagata, Taisuke Hasegawa \& Yuichiro Noiri

Division of Anatomy and Cell Biology of the Hard Tissue,

Department of Tissue Regeneration and Reconstruction, Niigata University Graduate School of Medical and Dental Sciences, 2-5274, Gakkocho-dori, Chuo-ku, Niigata, 951-8514, Japan

Hayato Ohshima

Division of Microbiology and Infectious Diseases, Niigata University Graduate School of Medical and Dental Sciences, 2-5274, Gakkocho-dori, Chuo-ku, Niigata, 951-8514, Japan

Yutaka Terao

\section{References}

1. Zimmer S, Nezhat V, Bizhang M, Seemann R, Barthel C. Clinical efficacy of a new sonic/ultrasonic toothbrush. J Clin Periodontol. 2002;29(6):496-500.

2. van der Weijden GA, Timmerman MF, Reijerse E, Snoek CM, van der Velden U. Comparison of 2 electric toothbrushes in plaque-removing ability. Professional and supervised brushing. $J$ Clin Periodontol. 1995;22(8):648-652.

3. Johnson BD, Mclnnes C. Clinical evaluation of the efficacy and safety of a new sonic toothbrush. $J$ Periodontol. 1994;65(7):692-697.

4. Emling RC, Yankell SL. The application of sonic technology to oral hygiene: the third generation of powered toothbrushes. J Clin Dent. 1997;8(1 Spec No):1-3.

5. Axelsson P, Albandar JM, Rams TE. Prevention and control of periodontal diseases in developing and industrialized nations. Periodontol 2000. 2002;29:235-246.

6. Jepsen S. The role of manual toothbrushes in effective dental biofilm control: Advantages and limitations. In: Lang NP, Attstrom R, Löe H, editors. Proceedings of the European Workshop on Mechanical Dental biofilm Control. London: Quintessence; 1998. pp 121-37.

7. Ak G, Sepet E, Pinar A, Aren G, Turan N. Reasons for early loss of primary molars. Oral Health Prev Dent. 2005;3(2):113-117.

8. Christou V, Timmerman MF, Van der Velden U, Van der Weijden FA. Comparison of different approaches of interdental oral hygiene: interdental brushes versus dental floss. $J$ Periodontol. 1998;69(7):759-764.

9. van der Weijden F, Slot DE. Oral hygiene in the prevention of periodontal diseases: the evidence. Periodontol 2000. 2011;55(1):104-123.

10. König J, Plagmann HC, Rühling A, Kocher T. Tooth loss and pocket probing depths in compliant periodontally treated patients: a retrospective analysis. J Clin Periodontol. 2002;29(12):1092-1100. 
11. Matuliene G, Studer R, Lang NP, et al. Significance of Periodontal Risk Assessment in the recurrence of periodontitis and tooth loss. J Clin Periodontol. 2010;37(2):191-199.

12. Adams H, Winston MT, Heersink J, Buckingham-Meyer KA, Costerton JW, Stoodley P. Development of a laboratory model to assess the removal of biofilm from interproximal spaces by powered tooth brushing. Am J Dent. 2002;15 Spec No:12B-17B.

13. Sharma PK, Gibcus MJ, van der Mei HC, Busscher HJ. Influence of fluid shear and microbubbles on bacterial detachment from a surface. Appl Environ Microbiol. 2005;71(7):3668-3673.

14. Tawakoli PN, Sauer B, Becker K, Buchalla W, Attin T. Interproximal biofilm removal by intervallic use of a sonic toothbrush compared to an oral irrigation system. BMC Oral Health. 2015;15:91.

15. Parini MR, Eggett DL, Pitt WG. Removal of Streptococcus mutans biofilm by bubbles. J Clin Periodontol. 2005;32(11):1151-1156.

16. Parini MR, Pitt WG. Dynamic removal of oral biofilms by bubbles. Colloids Surf B Biointerfaces. 2006;52(1):39-46.

17. Schmidt JC, Astasov-Frauenhoffer M, Waltimo T, Weiger R, Walter C: Influence of the amplitude of different side-to-side toothbrushes on noncontact biofilm removal. Clin Oral Investig 2019, 23(4):1951-1957.

18. Wakamatsu R, Takenaka S, Ohsumi T, Terao Y, Ohshima H, Okiji T. Penetration kinetics of four mouthrinses into Streptococcus mutans biofilms analyzed by direct time-lapse visualization. Clin Oral Investig. 2014;18(2):625-634.

19. Song L, Hou J, van der Mei HC, Veeregowda DH, Busscher HJ, Sjollema J: Antimicrobials Influence Bond Stiffness and Detachment of Oral Bacteria. J Dent Res 2016, 95(7):793-799.

20. Suzuki Y, Ohsumi T, Isono T, Nagata R, Hasegawa T, Takenaka S, Terao Y, Noiri Y: Effects of a subminimum inhibitory concentration of chlorhexidine gluconate on the development of. Biofouling 2020:1-13.

21. Ohsumi T, Takenaka S, Wakamatsu R, et al. Residual structure of Streptococcus mutans biofilm following complete disinfection favors secondary bacterial adhesion and biofilm re-development. PLoS One. 2015;10(1): e0116647.

22. Sedlacek MJ, Walker C. Antibiotic resistance in an in vitro subgingival biofilm model. Oral Microbiol Immunol. 2007;22(5):333-339.

23. Takenaka S, Oda M, Domon H, Ohsumi T, Suzuki Y, Ohshima H, Yamamoto H, Terao Y, Noiri Y: Vizantin inhibits bacterial adhesion without affecting bacterial growth and causes Streptococcus mutans biofilm to detach by altering its internal architecture. Biochem Biophys Res Commun 2016, 480(2):173-179.

24. Cotter JJ, O'Gara JP, Stewart PS, Pitts B, Casey E. Characterization of a modified rotating disk reactor for the cultivation of Staphylococcus epidermidis biofilm. J App/ Microbiol. 2010;109(6):2105-2117.

25. Rani SA, Pitts B, Beyenal H, et al. Spatial patterns of DNA replication, protein synthesis, and oxygen concentration within bacterial biofilms reveal diverse physiological states. J Bacteriol. 2007;189(11):4223-4233.

Page $12 / 19$ 
26. Tadokoro K, Yamaguchi T, Kawamura K, et al. Rapid quantification of periodontitis-related bacteria using a novel modification of Invader PLUS technologies. Microbiol Res. 2010;165(1):43-49.

27. Busscher HJ, Jager D, Finger G, Schaefer N, van der Mei HC. Energy transfer, volumetric expansion, and removal of oral biofilms by non-contact brushing. Eur J Oral Sci. 2010;118(2):177-182.

28. Stanford CM, Srikantha R, Wu CD. Efficacy of the Sonicare toothbrush fluid dynamic action on removal of human supragingival plaque. J Clin Dent. 1997;8(1 Spec No):10-14.

29. HOPE CK, PETRIE A, WILSON M (2003) In vitro assessment of the dental biofilm-removing ability of hydrodynamic shear forces produced beyond the bristles by 2 electric toothbrushes. J Periodontol 74(7), 1017-22.

30. van der Mei HC, Rustema-Abbing M, Bruinsma GM, Gottenbos B, Busscher HJ. Sequence of oral bacterial co-adhesion and non-contact brushing. J Dent Res. 2007;86(5):421-425.

31. Verkaik MJ, Busscher HJ, Rustema-Abbing M, Slomp AM, Abbas F, van der Mei HC. Oral biofilm models for mechanical plaque removal. Clin Oral Investig. 2010;14(4):403-409.

32. Hope CK, Petrie A, Wilson M. Efficacy of removal of sucrose-supplemented interproximal plaque by electric toothbrushes in an in vitro model. Appl Environ Microbiol. 2005;71(2):1114-1116.

33. YIN J, MEI ML, LI Q, XIA R, ZHANG Z, CHU CH $(2016)$ Self-cleaning and antibiofouling enamel surface by slippery liquid-infused technique. Sci rep2016; 6 doi: 10.1038/srep25924.

34. Bowen WH. Do we need to be concerned about dental caries in the coming millennium? Crit Rev Oral Biol Med. 2002;13(2):126-131.

35. Loesche WJ. Role of Streptococcus mutans in human dental decay. Microbiol Rev. 1986;50(4):353380.

36. Takenaka S, Trivedi HM, Corbin A, Pitts B, Stewart PS. Direct visualization of spatial and temporal patterns of antimicrobial action within model oral biofilms. Appl Environ Microbiol. 2008;74(6):18691875.

37. Sniehotta FF, Araújo Soares V, Dombrowski SU. Randomized controlled trial of a one-minute intervention changing oral self-care behavior. J Dent Res. 2007;86(7):641-645.

38. Leung WK, Ng DK, Jin L, Corbet EF. Tooth loss in treated periodontitis patients responsible for their supportive care arrangements. J Clin Periodontol. 2006;33(4):265-275.

39. Marsh PD. Oral ecology and its impact on oral microbial diversity. Oral bacterial ecology: the molecular basis 2000, Horizon Scientific Press; 2000. 11-65.

40. Marsh PD, Bradshaw DJ. Dental plaque as a biofilm. J Ind Microbiol. 1995;15(3):169-175.

\section{Figures}




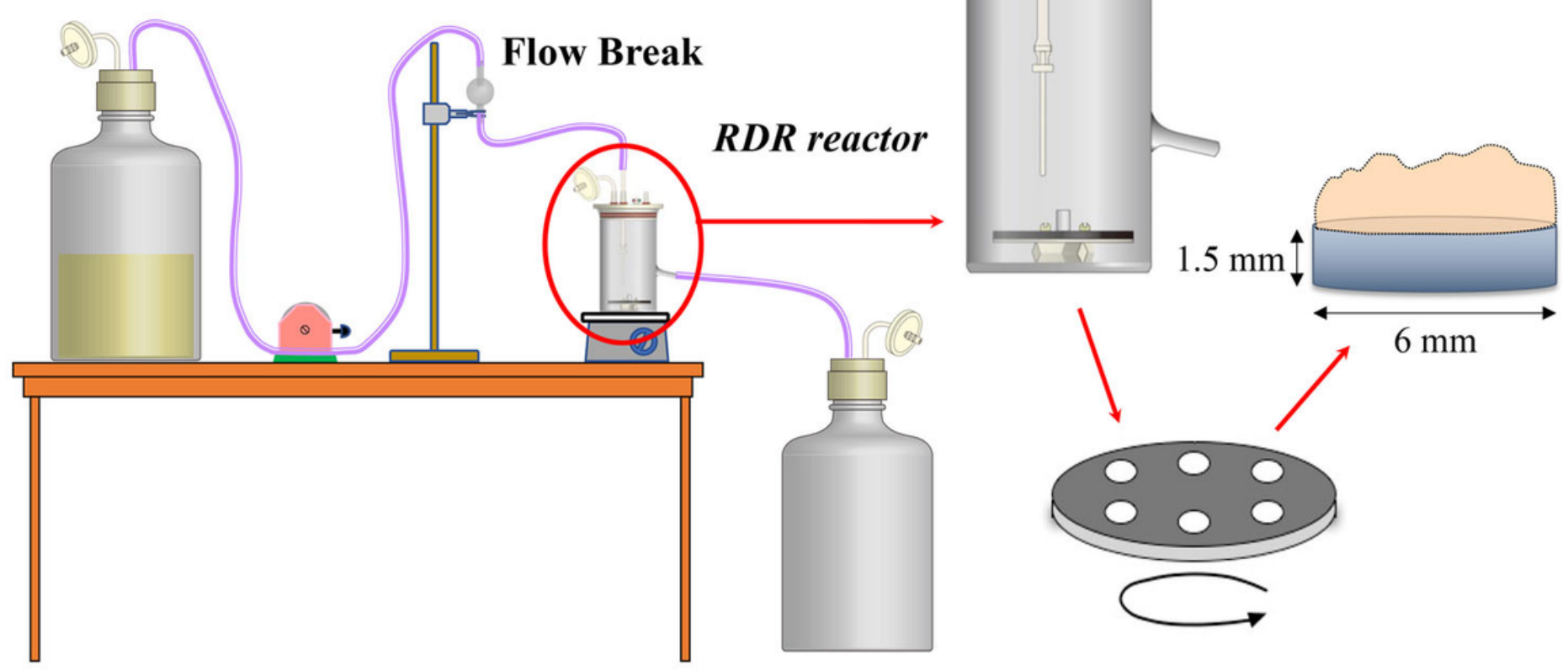

\section{Figure 1}

Schematic of the rotating-disc biofilm reactor system used to grow the Streptococcus mutans biofilms. The rotating-disc biofilm reactor is shown in the continuous flow mode of operation. The reactor was used to develop a standardized three-day S. mutans (ATCC 25175) biofilm on resin composite discs (Premise Flowable, Kerr). The size of the resin composite disc was standardized to $6 \mathrm{~mm}$ in diameter and $1.5 \mathrm{~mm}$ in thickness. The rotor was composed of a magnetic stir bar, on which a rotating-disc was attached to hold resin composite discs. The container equipped with the stirring bar was placed on a magnetic stirrer and incubated while rotating. Disc rotation provided continuous mixing of the solution added to the system. 

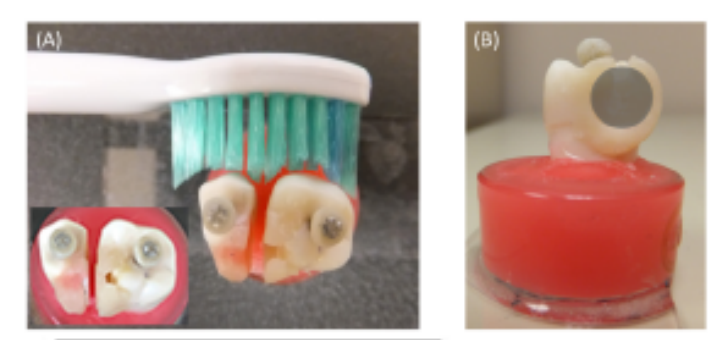

(C)

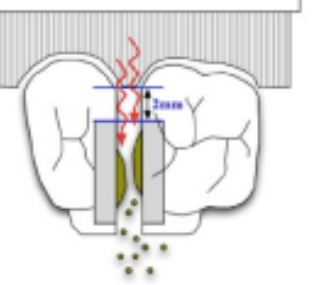

\section{Figure 2}

Interdental model of plastic teeth with mounted discs. The resin composite discs are separated from the reach of the toothbrush bristles by $2 \mathrm{~mm}$. The plastic teeth are positioned such that brushing is applied to the buccal surface (A). Inset: The discs are held perpendicular to the plane of the brushing action, the gap mimicking the embrasure. The approximate position of the toothbrush is shown in (B). Oral devices are positioned perpendicularly to the fixed specimens, as illustrated in (C) for the sonic toothbrush. A gap was provided to ensure that the tip of the toothbrush did not hit the tooth, and the position of the electric toothbrush was set. 


\section{dense area}

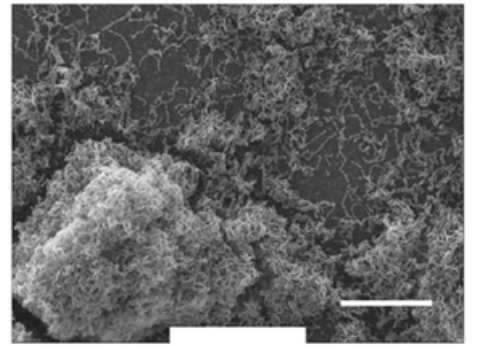

$\mathrm{C}$

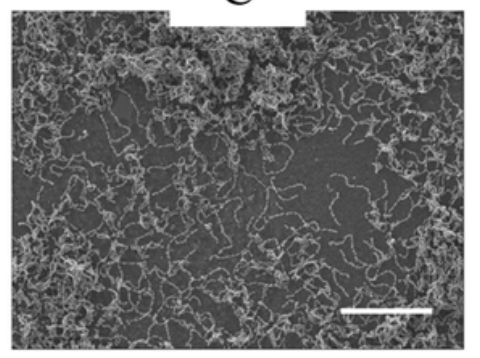

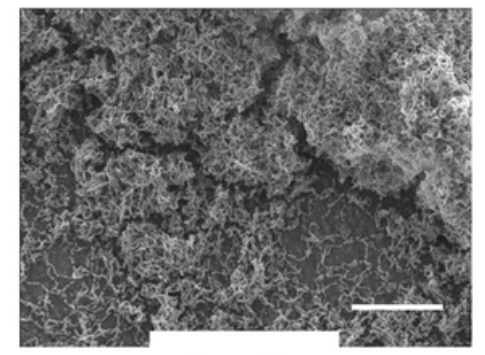

$\mathrm{C}+\mathrm{ST}$

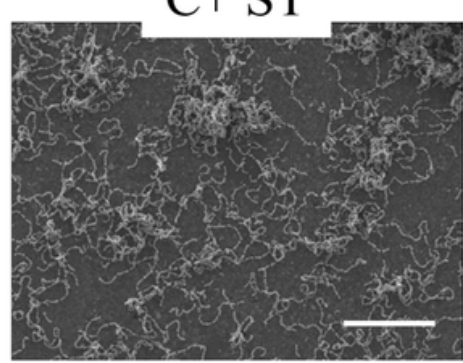

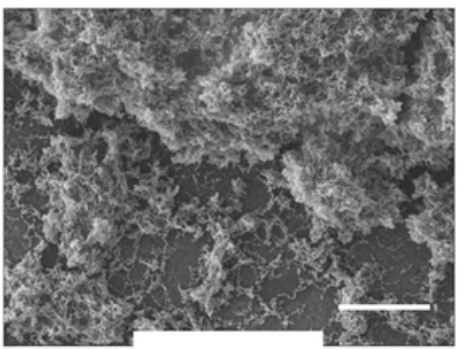

$\mathrm{G}+\mathrm{ST}$

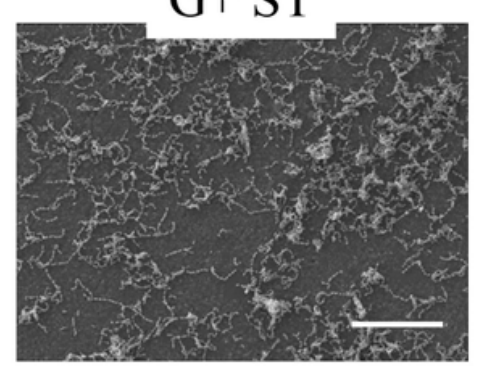

sparse area
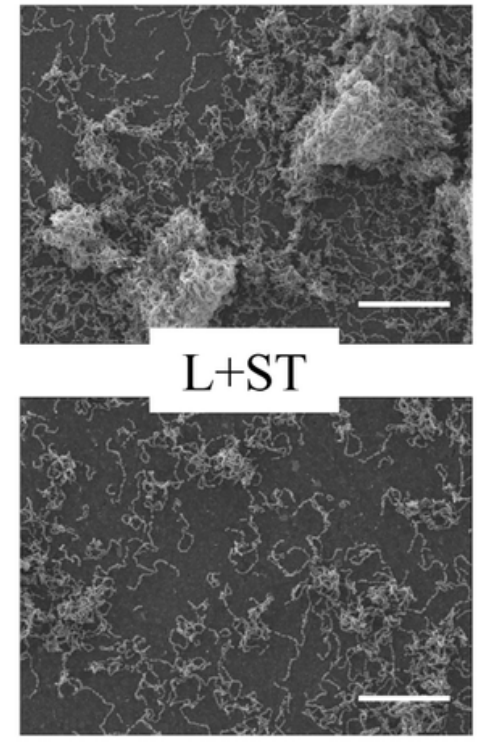
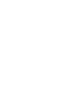

\section{Figure 3}

SEM images of the biofilm structure remaining on the disc after each experimental treatment $(C, C+S T, G$ $+S T$, and $L+S T$ ). The upper row depicts representative SEM images of the dense area of the biofilm structure in each experimental group. The lower row shows representative SEM images of the sparse area of the biofilm structure in each experimental group. Scale bar, $20 \mu \mathrm{m}$. 


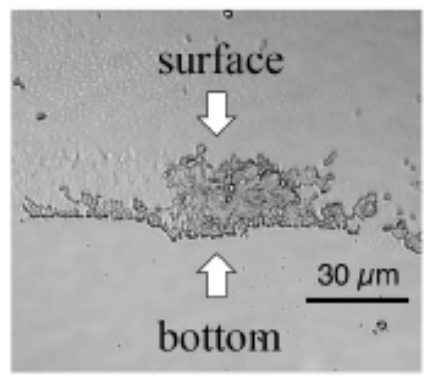

\begin{tabular}{|c|l|}
\hline $\mathrm{C}$ & $28.43 \pm 9.31^{\mathrm{a}}$ \\
\hline $\mathrm{C}+\mathrm{ST}$ & $26.81 \pm 4.61^{\mathrm{a}}$ \\
\hline $\mathrm{G}+\mathrm{ST}$ & $23.10 \pm 4.71^{\mathrm{a}}{ }^{\mathrm{b}}$ \\
\hline $\mathrm{L}+\mathrm{ST}$ & $15.86 \pm 6.25^{\mathrm{a}}$ \\
\hline
\end{tabular}

\section{Figure 4}

Comparison of the thickness of the biofilm structure between the control group (C) and the sonic toothbrush combined groups $(C+S T, G+S T$, and $L+S T)$. The left panel shows a representative transmission image of a frozen longitudinal section of the biofilm (scale bar, $30 \mu \mathrm{m}$ ). The biofilm thickness was measured from the bottom surface of the biofilm to the top surface of the biofilm. The thickest parts were measured for each section. Data are presented as means $\pm S D(n=25$, Steel-Dwass test). Same alphabetic character indicates that values are not significantly different $(p>0.05)$. 


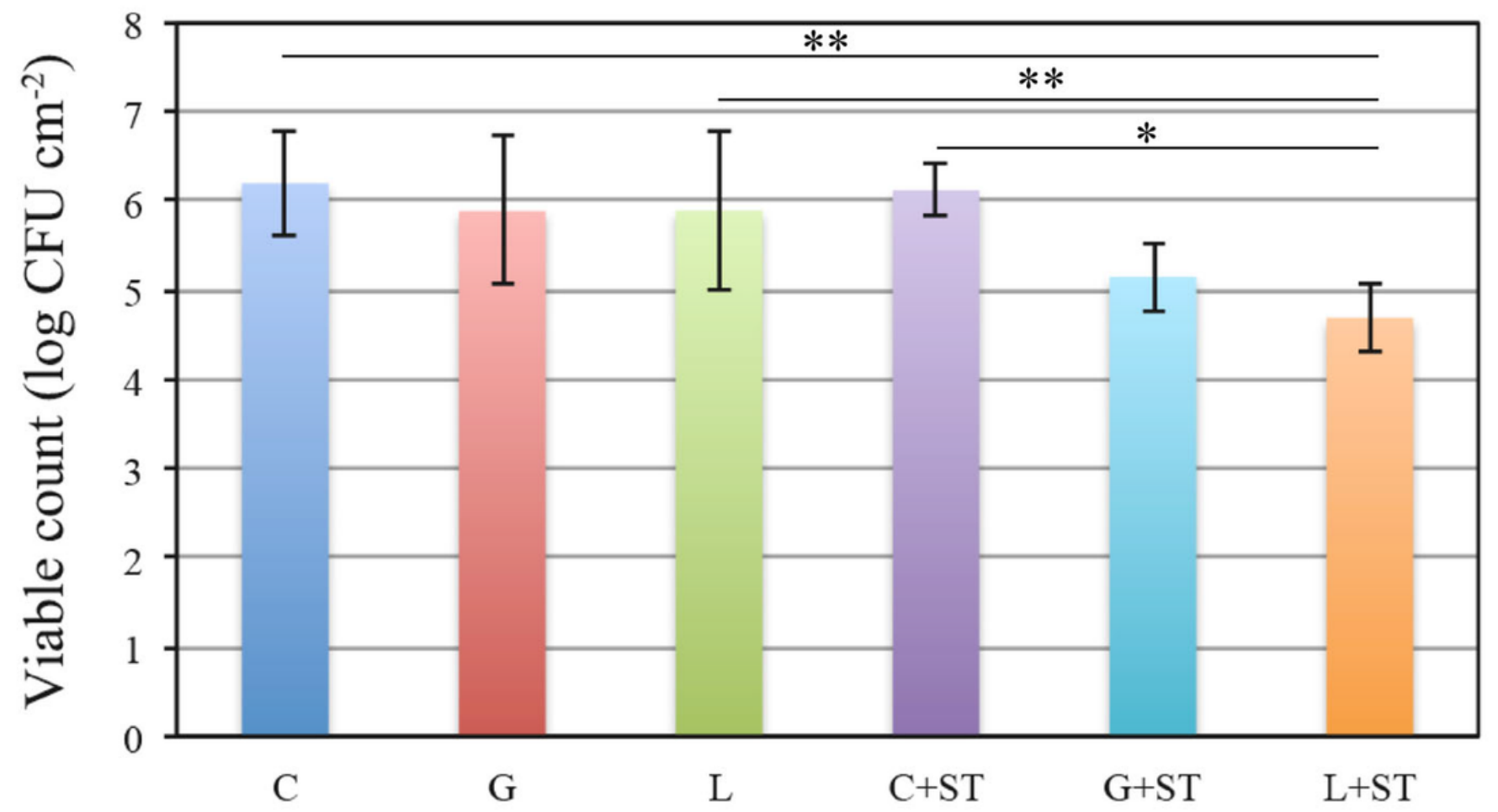

Figure 5

Calculation of the number of live cells remaining on the resin discs after treatment for 5 seconds. Colonies were counted and the bacterial load was calculated. The bacterial load was assessed as the number of bacteria in colony forming units (CFU) per $\mathrm{cm}-2$ (corresponding to the unit area of one resin disc). Data are presented as means \pm SD $(n=7$, one-way ANOVA, Steel-Dwass test * $p<0.05$ and $* * p<$ 0.01). Error bars represent $95 \%$ confidence intervals. 


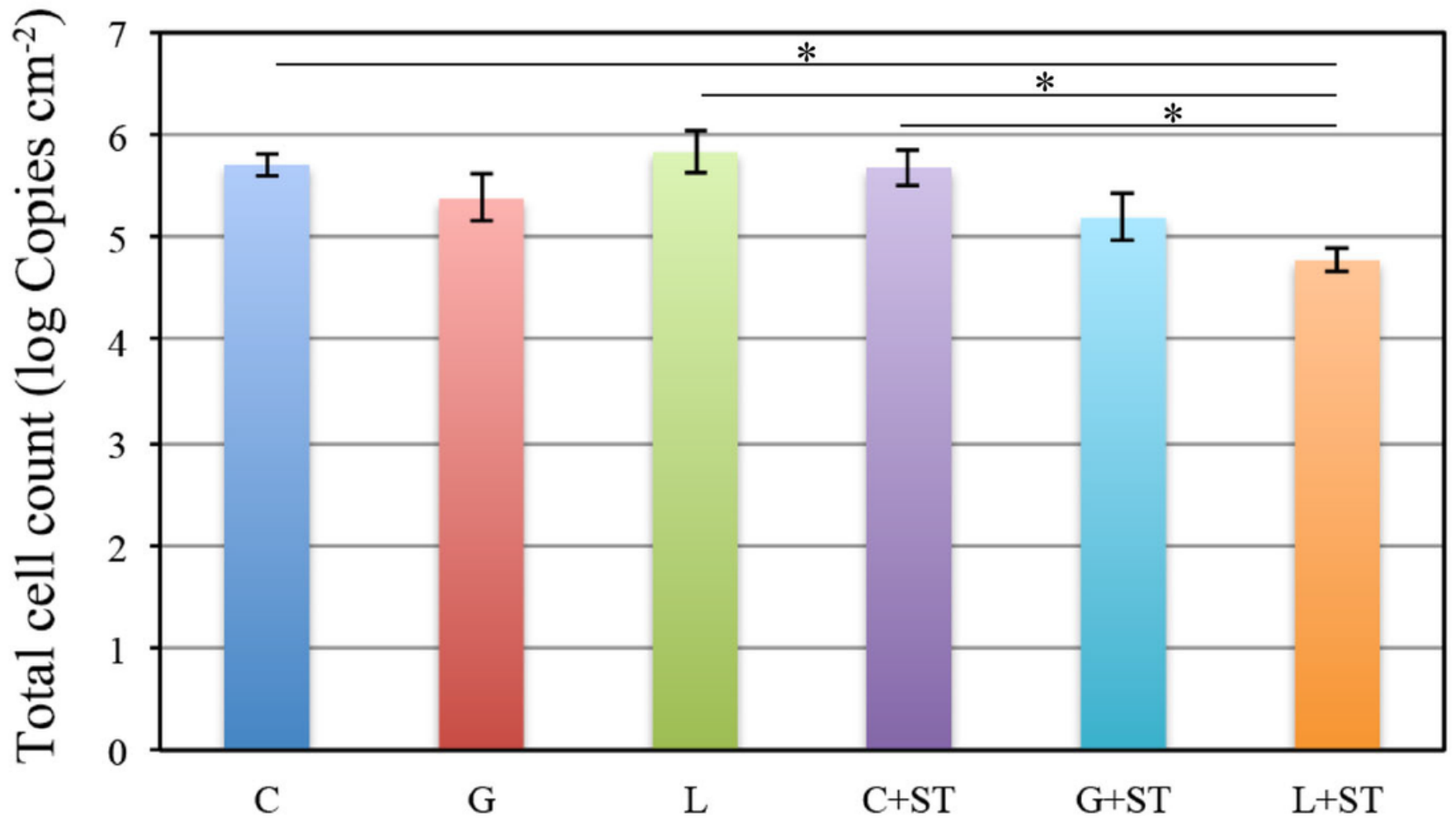

Figure 6

Total number of $\mathrm{S}$. mutans cells present in the biofilm remaining on the resin discs after an immersion and a combination treatment for 5 seconds. The total bacterial load was assessed as the number of bacteria determined using the PCR-invader assay per $\mathrm{cm}-2$ (corresponding to the unit area of one resin disc). Data are presented as means \pm SD $(n=7$, one-way ANOVA, Steel-Dwass test * $p<0.05)$. Error bars represent $95 \%$ confidence intervals. 